\title{
HEXACO Personality and Schwartz's Personal Values: A Facet-Level Analysis
}

\author{
Jeromy Anglim, Emily R. V. Knowles, Patrick D. Dunlop, Andrew \\ Marty ${ }^{1}$
}

\begin{abstract}
This study systematically examined the correlates of Schwartz's basic values with the broad and narrow traits of the HEXACO model of personality. A sample of 1244 adults (53\% male; $M$ age $=44, S D=12$ ) completed the 200-item HEXACO-PI-R and the Portrait Values Questionnaire measuring Schwartz's 10 basic personal values. Regression models predicting each of the ten basic values from personality revealed mean-adjusted multiple correlations of .39 for HEXACO factors without honesty-humility, .45 for all HEXACO factors, and .53 for HEXACO facets. The facet-level multiple correlations were particularly large $(>.60)$ for power, universalism, and cooperation. Results suggest that individual differences in personality and values overlap to a greater extent than implied by past literature.
\end{abstract}

Keywords. personality, HEXACO, values, narrow traits, facets

\section{Introduction}

Personality traits and personal values both represent broad constructs used to characterize fundamental individual differences (Parks-Leduc, Feldman, \& Bardi, 2015; Roccas, Sagiv, Schwartz, \& Knafo, 2002). Whereas a person's personality represents their pattern of behaviors, thoughts and emotions (McCrae \& Costa, 1987), a person's values represent broad evaluative judgments about what is good or important in life (Bilsky \& Schwartz, 1994; Schwartz, 1992; Vernon \& Allport, 1931). The two constructs are distinct but interrelated and have been mapped onto one another in various ways in the psychological literature (Blickle, Schlegel, Fassbender, \& Klein, 2006; Lee, Ashton,

\section{${ }^{1}$ Citation Information:}

Anglim, J., Knowles, E. R. V., Dunlop, P. D., \& Marty, A. (2017). HEXACO Personality and Schwartz's Personal Values: A Facet-Level Analysis. Journal of Research in Personality, 68, 23-31. http://dx.doi.org/10.1016/j.jrp.2017.04.002 Please see the doi to publisher's copy of record.

Jeromy Anglim and Emily R. V. Knowles, School of Psychology, Deakin University, Geelong, Australia; Patrick D. Dunlop, School of Psychological Science, The University of Western Australia (M304), 35 Stirling Highway, Crawley, Western Australia, Australia, 6009; Andrew Marty, SACS Consulting, 350 Collins Street, Melbourne, Victoria, Australia, 3000. Data, reproducible analysis scripts, and materials are available at https://osf.io/wkc5u "HEXACO Personality and Schwartz's Personal Values: A Facet-Level Analysis - Open Science Framework". Correspondence concerning this article should be addressed to Jeromy Anglim, School of Psychology, Deakin University, Locked Bag 20000, Geelong, 3220 Australia. Email: jeromy.anglim@deakin.edu.au 
Ogunfowora, Bourdage, \& Shin, 2010; Lee et al., 2009; Luk \& Bond, 1993; Olver \& Mooradian, 2003; Parks-Leduc et al., 2015; Pozzebon \& Ashton, 2009; Roccas et al., 2002; Saroglou \& Muñoz-García, 2008). However, most of this research has focused on the "Big 5" personality factors of neuroticism, extraversion, openness, agreeableness, and conscientiousness, whereas there is considerable evidence from other areas that narrower personality facets can provide a more nuanced understanding of how personality covaries with criteria of interest (Anglim \& Grant, 2016; Paunonen \& Jackson, 2000). Furthermore, the relatively recent introduction of the HEXACO model of personality, which incorporates the honesty-humility factor, has raised several questions about the status of this factor within the combined conceptual space that includes personality traits and personal values. Thus, in this study, we examine how broad and narrow personality traits, using the HEXACO framework of personality, relate to personal values in a large community sample of Australian adults.

\section{Definitions and Frameworks of Personality Traits and Values}

Personality traits describe people's characteristic ways of thinking, feeling, and behaving. Personality traits are typically organized in a hierarchical framework with broad personality factors composed of narrower personality facets. While the Big 5 model (i.e., openness, conscientiousness, extraversion, agreeableness, and neuroticism, Costa Jr $\&$ McCrae, 1995) has served a useful synthesizing role in the personality literature, researchers have observed several shortcomings (Paunonen \& Jackson, 2000). In particular, several researchers have argued that a sixfactor model of personality is more robust than the Big 5 across languages and cultures (Ashton et al., 2004; Lee \& Ashton, 2008). The six-factor personality framework has come to be called HEXACO, with its name being an acronym formed by its factors, namely, honestyhumility, emotionality, extraversion, agreeableness, conscientiousness, and openness to experience (Lee \& Ashton, 2004). In addition to reconfiguring Big 5 neuroticism and agreeableness into HEXACO emotionality and agreeableness (for a discussion, see Ashton, Lee, \& de Vries, 2014), the HEXACO model introduces an honesty-humility factor. This factor is demonstrably important for the prediction of certain criteria-typically those that fall within the spectrum of anti-social behaviors - with HEXACO offering substantial incremental validity beyond the Big 5 (e.g., Lee, Ashton, Morrison, Cordery, \& Dunlop, 2008; Lee, Gizzarone, \& Ashton, 2003). In this research, we adopt the HEXACO model as, for reasons we articulate below, we believe the honesty-humility factor provides important insight into understanding values.

Most factor-based personality frameworks adopt a hierarchical approach whereby narrower traits are nested within, and help to define, the broader factors. Various frameworks have been proposed, including the NEO-PI-R with six facets for its five factors, (Costa Jr \& McCrae, 
1995), the 10 aspects of the Big 5 by DeYoung, Quilty, and Peterson (2007), and the HEXACO model of personality that includes four facets for each of its six factors (Ashton et al., 2014), plus a $25^{\text {th }}$ interstitial facet, altruism. Despite the lack of agreement regarding the structure of personality at the facet-level, a more general debate in the literature concerns whether facets are needed (Anglim \& Grant, 2014; Paunonen \& Ashton, 2001; Paunonen \& Jackson, 2000). While some researchers have taken more extreme positions that broad factors are all that is required or that using narrow facets can lead to substantial incremental prediction, a more common finding is that narrow traits lead to modest but meaningful incremental prediction (Anglim \& Grant, 2016; Horwood, Anglim, \& Tooley, 2015; Paunonen \& Ashton, 2001; Paunonen, Haddock, Forsterling, \& Keinonen, 2003). Furthermore, a general proposition is that broad traits best predict broad criteria and narrow traits best predict narrow criteria (Ones \& Viswesvaran, 1996).

Personal values are broad evaluative criteria that provide a framework for prioritizing goals and determining what is important or good in life (Bilsky \& Schwartz, 1994; Rokeach, 1973; Schwartz, 1992). Various taxonomies of values have been proposed, but the framework proposed by Schwartz is arguably the most established in psychology (Schwartz, 1992; Schwartz et al., 2012). Schwartz proposed that there are 10 basic values that are largely generalizable across cultures. These are represented clockwise in circular space as self-direction, stimulation, hedonism, achievement, power, security, tradition, conformity, benevolence, and universalism. The ten values are arranged in a circular motivational continuum where values closer on the circle are assumed to be similar and values on opposite sides tend to be negatively related (Schwartz et al., 2012). This circular arrangement of ten values also includes concepts representing various regions of the value-space including self-transcendence versus self-enhancement and conservation versus openness to change.

\section{Conceptual and Empirical Relationships between Personality and Values}

Several clear conceptual distinctions can be made between personality traits and values (Parks-Leduc et al., 2015). First, traits are descriptions of behavioral, cognitive, and affective tendencies, whereas values describe broad motivations or goals. For example, people high on the trait of extraversion might be the life of the party, whereas a person can value being outgoing in social gatherings, independently of whether they are prone to acting in a lively or sociable manner. Second, some researchers theorize that traits are more biologically based whereas values are more culturally based (for a review, see Parks-Leduc et al., 2015). Similarly, there is a tradition of attempting to separate traits and values, whereby traits represent temperament and values represent character (Allport, 1937). Third, values are always cognitive whereas traits are not necessarily cognitive. Theoretically, some view both personality and values as elements of a broader concept of total 
personality, but within different frameworks (Parks-Leduc et al., 2015), and others view personality as primary and values as secondary (Rokeach, 1973; Vecchione, Alessandri, Barbaranelli, \& Caprara, 2011). Some researchers argue that there are dynamic, reinforcing, and reciprocal effects between personality traits and values, however personality and values are not one and the same (Parks-Leduc et al., 2015; Roccas et al., 2002).

Big 5 and Schwartz's Values

Most studies that empirically examine the relations between personality and values have measured Big 5 personality traits and Schwartz's personal values (e.g., Haslam, Whelan, \& Bastian, 2009; Parks-Leduc et al., 2015; Roccas et al., 2002). This research has shown that there is meaningful overlap between personality and values. Indeed, a recent meta-analysis by Parks-Leduc et al. (2015) provides an overview of the empirical relationships between the Big 5 and values. To summarize and synthesize these results, we present these meta-analytic correlations (uncorrected) in Table 1 along with calculations of the mean absolute correlations. In particular, we note that the mean absolute correlation for each personality factor is informative in identifying which personality factors overlap relatively more with values. As Table 1 shows, while the average absolute Big 5-value correlation is small (i.e., .12 ), many absolute correlations are greater than .20 (12 out of 50), and three are larger than .30 : agreeableness with power $(-.31)$, agreeableness with benevolence (.45), and openness with self-direction (.37). Of the Big 5 , openness shows the largest number of absolute correlations greater than .20 (i.e., five), but extraversion and agreeableness also exhibit average absolute correlations that are arguably not much lower than those of openness. Thus, overall, at the factor level, openness, agreeableness, and extraversion show appreciable relations with Schwartz's values but conscientiousness and, particularly, emotional stability appear largely uncorrelated with values.

Only a few studies we are aware of have examined the relations between narrow facets of the Big 5 and Schwartz's 10 values (i.e., Dollinger, Leong, \& Ulicni, 1996; Roccas et al., 2002; Saroglou \& Muñoz-García, 2008). In one study, Dollinger et al. (1996) used an early version of the NEO that included facets for only three factors and only briefly commented on the correlations. Two studies (Roccas et al., 2002; Saroglou \& Muñoz-García, 2008) revealed correlations of the full set of NEO facets $(n=246, n=256)$ with values in the context of predicting religious orientation. Roccas et al. (2002) observed a few differential patterns of facet correlations with particular values. For instance, the conscientiousness facets of competence, achievement striving, and selfdiscipline correlated more strongly with achievement, whereas the facets of order and dutifulness correlated more strongly with conservatism. 
Table 1

Summary of Mean Raw Correlations between Big 5 and Schwartz Personal Values from ParksLeduc et al. (2015) Meta-Analysis

\begin{tabular}{lccccccccccc}
\hline & SD & ST & HE & AC & PO & SE & CO & TR & BE & UN & Mean Abs. $r$ \\
\hline Emotional stability & -.01 & .01 & .01 & -.01 & .02 & -.02 & -.04 & -.02 & -.01 & -.03 & .02 \\
Extraversion & .12 & .28 & .16 & .23 & .23 & -.04 & -.13 & -.18 & -.04 & -.05 & .15 \\
Agreeableness & -.04 & -.04 & -.08 & -.18 & -.31 & .00 & .18 & .15 & .45 & .29 & .17 \\
Conscientiousness & .01 & -.12 & -.15 & .12 & .04 & .27 & .20 & .07 & .05 & -.01 & .10 \\
Openness & .37 & .27 & .07 & .08 & -.04 & -.17 & -.20 & -.21 & .10 & .28 & .18 \\
Mean Absolute $r$ & .11 & .14 & .09 & .12 & .13 & .10 & .15 & .13 & .13 & .13 & .12 \\
\hline
\end{tabular}

Note. Mean correlations are based on between 51 and 56 studies and between 53,692 and 55,072 cases. Absolute correlations greater than or equal to .20 are bolded. Mean Abs. $r$ is the mean absolute correlation for the corresponding row or column. $\mathrm{SD}=$ Self-direction, $\mathrm{ST}=\mathrm{Stimulation}$, $\mathrm{HE}=$ Hedonism, $\mathrm{AC}=$ Achievement, $\mathrm{PO}=$ Power, $\mathrm{SE}=$ Security, $\mathrm{CO}=$ Conformity, $\mathrm{TR}=$ Tradition, $\mathrm{BE}=$ Benevolence, $\mathrm{UN}=$ Universalism.

\section{HEXACO and Schwartz's Values}

To the best of our knowledge, only a small number of studies have measured both HEXACO and Schwartz's personal values (i.e., Lee et al., 2010; Lee et al., 2009; Pozzebon \& Ashton, 2009). Pozzebon (2006) perhaps provides the most comprehensive examination to date. A subset of the results are presented in Pozzebon (2006) were later analyzed by Pozzebon and Ashton (2009) who compared prediction of behavior using HEXACO factors and Schwartz's 10 values. Lee et al. (2010) also report some data linking HEXACO factors and the broad values of conservation and self-enhancement within the context of examining correlations of personality with political values. In the context of a paper on self-other agreement, Lee et al. (2009) also reported results from three datasets that included HEXACO facets and Schwartz values: (a) a university sample $(n=245)$ using a half-length version of the HEXACO Personality Inventory (HEXACO-PI) (Pozzebon, 2006), (b) a second university sample using the full-length revised HEXACO-PI (HEXACO-PI-R), and (c) Goldberg's Oregon community sample $(n=$ 673) using the HEXACO-PI. They presented selected correlations between personality facets and two broad values based on factor analysis of the Schwartz's 10 basic values (i.e., self-transcendence vs. selfenhancement and openness to change vs. conservation). They also commented on a few stand-out correlations of HEXACO facets with the 10 basic values (e.g., the honesty-humility facet of greed avoidance with power, $r=-.50$ and the openness facet of aesthetic appreciation with selfdirection, $r=.44$ ). A major conclusion was that two broad factors of values were principally associated with honesty-humility and openness to 
experience. They concluded that the relation between HEXACO and the two broad values factors was not caused by unusually strong correlations of particular facets.

In order to synthesize current understanding, Table 2 presents the only published correlations between HEXACO and the 10 personal values, drawn from Pozzebon (2006) based on a sample of 252 participants, along with mean absolute correlations. Although based on a modest sample size, several patterns emerged. First, many of the relations present were similar to those seen in the Parks-Leduc et al. (2015) metaanalysis, particularly for openness and agreeableness. Second, correlations of HEXACO emotionality with values are somewhat larger than those of Big 5 neuroticism, which is likely partly explained by reconfiguration of agreeableness and emotionality in the HEXACO model, relative to Big 5 agreeableness and emotional stability. Third, honesty-humility had the strongest overall mean absolute correlation with values.

Table 2

Correlations between HEXACO (100 item) and Schwartz Personal Values from Pozzebon (2006)

\begin{tabular}{lccccccccccc}
\hline & SD & ST & HE & AC & PO & SE & CO & TR & BE & UN & Mean Abs. $r$ \\
\hline Honesty-Humility & .08 & -.12 & $\mathbf{- . 3 0}$ & $\mathbf{- . 2 8}$ & $\mathbf{- . 5 3}$ & -.06 & .16 & $\mathbf{. 2 3}$ & $\mathbf{. 4 5}$ & $\mathbf{. 3 6}$ &. $\mathbf{2 6}$ \\
Emotionality & -.13 & $\mathbf{- . 3 4}$ & $\mathbf{- . 2 2}$ & -.05 & -.23 & .11 & .09 & .06 & .18 & .05 & .15 \\
Extraversion &. $\mathbf{2 0}$ & .09 & -.06 & .09 & -.02 & -.11 & .06 & -.12 & .03 & -.11 & .09 \\
Agreeableness & -.08 & .10 & -.10 & -.27 & -.34 & -.06 & .10 &. $\mathbf{2 8}$ &. $\mathbf{2 4}$ & .18 & .18 \\
Conscientiousness & -.15 & $\mathbf{- . 3 0}$ & -.19 & $\mathbf{. 3 0}$ & .06 & .10 & $\mathbf{. 3 2}$ & .12 & -.01 & -.09 & .16 \\
Openness & $\mathbf{. 3 8}$ & .06 & $\mathbf{- . 2 2}$ & -.14 & $\mathbf{- . 2 0}$ & -.28 & -.07 & -.11 & .01 & $\mathbf{. 4 2}$ & .19 \\
& & & & & & & & & & & \\
Mean Absolute $r$ & .17 & .17 & .18 & .19 & $\mathbf{. 2 3}$ & .12 & .13 & .15 & .15 & .20 & .17 \\
\hline
\end{tabular}

Note. $N=252$. Correlations larger than .20 are bolded. Mean Abs. $r$ is the mean absolute correlation for the corresponding row or column.

$\mathrm{SD}=$ Self-direction, $\mathrm{ST}=$ Stimulation, $\mathrm{HE}=$ Hedonism, $\mathrm{AC}=$ Achievement, $\mathrm{PO}=$ Power, $\mathrm{SE}=$ Security, $\mathrm{CO}=$ Conformity, $\mathrm{TR}=$ Tradition, $\mathrm{BE}=$ Benevolence, $\mathrm{UN}=$ Universalism.

\section{The Current Study}

As discussed, most literature comparing personality traits and personal values has focused on the Big 5. Very few studies have examined the relations of personality facets and values, and most of these studies have simply reported zero-order correlations, and have not explored more parsimonious and systematic methods for assessing the incremental prediction of narrow facets over factors. Additionally, these studies have also often relied on modest sample sizes of fewer than 300 cases, which create challenges given the multiplicities inherent in examining the incremental validity of personality facets. Research 
relating values to HEXACO personality is also limited to only a few studies (i.e., Lee et al., 2010; Lee et al., 2009; Pozzebon \& Ashton, 2009), none of which has yet provided a systematic and comprehensive mapping of the HEXACO facets with Schwartz's personal values. The mapping of HEXACO and personal values is particularly important given the relevance of honesty-humility.

The present study therefore aimed to investigate the relation of HEXACO personality factors and facets with Schwartz's personal values. To achieve this aim, the factors and facets of HEXACO-PI-R in addition to Schwartz's Personal Values Questionnaire were measured in a large community sample. Correlations between personality traits and values were estimated along with regression models estimating the incremental predictive validity of narrow facets over broad factors.

\section{Method}

Data, reproducible analysis scripts, and materials are available at https://osf.io/wkc5u.

\section{Participants and Procedure}

Participants were recruited from a large database of email contacts maintained by an Australian professional consulting organization. Prospective participants were emailed an invitation to complete the research questionnaire. They were assured that their responses would remain confidential and only be used for research purposes. As an incentive to complete the survey, participants were given the opportunity to be randomly selected to receive a substantial travel voucher. The questionnaires consisted of measures of personality and values followed by basic demographics. After removing four cases due to random responding, the final sample consisted of 1244 participants (53\% male) with ages ranging from 18 to 70 years $(\mathrm{M}=44.3, S D=11.8)$. Almost all participants resided in Australia.

While in practical terms, the sample size was determined by the proportion of participants in the database who chose to complete the study, we note that large sample sizes are particularly important for studies that seek to map facet-level personality onto other variables such as values. With the current sample size, the standard error of correlations is less than .03. Such precision is particularly helpful when making subtle comparisons of the relative overlap of different traits and values. A large sample size is also important when estimating the incremental prediction of outcomes from facets (e.g., 25 predictors) versus domains (e.g., 6 predictors). Indeed, based on some reasonable assumptions of incremental prediction, one simulation study by Anglim and Grant (2014) showed that the standard error of incremental variance explained by 30 facets over 5 domains was .041 for $n=200$, and .016 for $n=1000$. Thus, sample sizes greater than 1000 provide a strong basis for obtaining refined estimates of incremental prediction by facets over domains. 


\section{Measures}

Personality

Personality traits were measured using the 200-item version of the HEXACO PI-R (Ashton et al., 2014; Lee \& Ashton, 2004, 2006). The questionnaire measures the six broad HEXACO factors and 25 narrow facets (i.e., four facets per factor and the interstitial facet of altruism). Each facet was measured using eight items, comprising a mix of positive and negatively keyed items. Responses were collected on a scale from 1 $=$ strongly disagree to $5=$ strongly agree. Observed factors and facet scores were obtained as the mean of constituent items after relevant item key reversal. We also examined the degree to which facets correlated with values after controlling for the six broad factors. To examine this, we obtained residualized facet scores by saving the residuals resulting from a linear regression of a given facet on the six HEXACO factors (Anglim \& Grant, 2014).

Values

Schwartz's values were measured using the Portrait Values Questionnaire 5X (PVQ) (Schwartz et al., 2012). The questionnaire consists of 57 positively-keyed items and yields scores for each of 10 basic values. Although we focused on the 10 basic values, some values can be decomposed into two or three narrower "refined" values (e.g., the basic value of power can be decomposed into the refined values of dominance and resources). Each refined value is measured by three items. Thus, each basic value is measured by three, six, or nine items, depending on the number of refined values which comprise the basic value. Each item of the PVQ adopts a third-person orientation describing a person of the same gender as the respondent. An example item phrased for a male respondent is: "It is important to him to have all sorts of new experiences". Participants rated each value on a scale from $1=$ [this is] opposed to my principles to $6=[$ this is] of great importance. Schwartz, Caprara, and Vecchione (2010) report test-retest correlations for the PVQ measure of values $(n=1030)$ ranging from $r=.65$ (benevolence) to .75 (achievement and hedonism).

Consistent with common practice when using the PVQ scale, we adopted an ipsatization procedure to obtain scores for each of the 10 values (Parks-Leduc et al., 2015; Schwartz et al., 2012). The logic of ipsatizing scores is that, in practice, people often need to make trade-offs between values when choosing concrete goals and courses of action; that is, they cannot prioritize all values simultaneously, even though they might rate each value as being important in absolute terms when completing the PVQ. In general, ipsatization is any method that ensures that the sum of scale scores is equal across individuals (Baron, 1996). Here, ipsatization was achieved using the deviational ipsative approach (Meade, 2004) or what is sometimes called within-person centering. This involved the following steps. First, raw (non-ipsatized) 'scale scores' for the 10 basic values were calculated for each participant as the mean 
response to the corresponding scale items. Second, an overall mean raw score was obtained for each participant as the mean response to all raw SVQ scales. Thus, this overall raw mean measures a general tendency to rate values as important. Third, the overall raw mean was subtracted from the raw non-ipsatized scale score to obtain an ipsatized scale score.

As a robustness check, we also performed an alternative ipsatization approach where residuals from a regression predicting scale scores from total scores were saved. We then inspected the correlations between the two sets of ipsatized scores; these correlations were all greater than .95 and so we were confident that the method of ipsatization we chose was unlikely to affect substantive findings.

\section{Data Analytic Approach}

The analyses sought to examine both the overall prediction of the 10 basic values from the broad HEXACO factors (with and without honesty-humility) and the narrow HEXACO facets, as well as examine the specific correlations between HEXACO personality traits and the 10 basic values. Comparisons between regression models were undertaken by comparing adjusted multiple $\mathrm{R}$ (i.e., the square root of adjusted Rsquared). Taking the square root of adjusted R-squared puts prediction on a correlational metric, and allows for clearer comparison of regression models with bivariate correlations. So, for example, it is easy to compare the correlation between the single factor of honesty-humility and universalism with the adjusted multiple $\mathrm{R}$ of a regression with all HEXACO factors predicting universalism. Using adjusted R-squared rather than raw R-squared as the basis for the multiple correlation is particularly important when examining the multiple correlation of narrow personality traits with criteria, given the large differences in number of predictors in factor versus facet regression equations (Anglim \& Grant, 2014). Both raw $R_{\text {adj }}^{\text {(facets) }}-R_{\text {adj }}^{\text {(factors }}$ and proportional $\left(R_{\text {adj }}^{\text {(facets) }}-R_{\text {adj }}^{\text {(factors) }}\right) / R_{\text {adj }}^{\text {(factors) }}$ increases in adjusted multiple $R$ (i.e., square root of adjusted $R$-squared) explained by facets over factors were computed.

When comparing the correlations between facets and values, we examined correlations with values using both observed and residualized personality facet scores. Correlations with residualized scores correspond to semi-partial correlations between facets and values, where overlap between factors and facets are controlled for. Whereas zero-order correlations provide an intuitive overview of how facets correlate with values, semi-partial correlations examine specifically what is unique about facet-value correlations. Given the large number of facet-value combinations (10 values by 25 facets $=250$ cross-correlations), we applied a conservative statistical significance criterion of $p<.001$. In addition to reporting correlations between traits and values, we also included the mean absolute cross-correlation for each value and each 
trait. This is particularly important in highlighting which traits overlap more with values, and which values overlap more with personality.

\section{Results}

\section{Descriptive Statistics}

Means, standard deviations, internal consistency of HEXACO factors, HEXACO facets, and Schwartz's basic values are provided in the online supplement along with intercorrelations. The mean Cronbach's alpha was .89 (range: .87 to .92 ) for HEXACO factors, .79 (range: .67 to .86 ) for HEXACO facets, and .76 (range: .63 to .84) for personal values. Exploratory factor analysis of the 25 personality facets showed strong alignment to the theorized structure, and a multidimensional scaling analysis of the basic values showed good support for the theorized circular arrangement of values (see online supplement for details).

\section{Factor correlations}

Table 3 presents the zero-order correlations of the HEXACO factors with Schwartz's 10 values along with the mean absolute correlations observed for each construct. The mean absolute crosscorrelation was .16. This value is statistically significant and much larger than the value of .03 we obtained from a simulation with randomly generated data of the same structure (see the rand.test function in the multicon package, Sherman \& Funder, 2009). Honesty-humility and openness exhibited the largest average absolute correlations, followed by agreeableness, extraversion, conscientiousness, and emotionality. While honesty-humility shared the most variance with values, its mean absolute correlation was only slightly larger than for openness. In general, correlations of honesty-humility with values tended to align with the axis of the personal values motivational continuum corresponding to selftranscendence (i.e., positive correlations with universalism and benevolence) versus self-enhancement (i.e., negative correlations with hedonism, achievement, and power). In contrast, openness correlated highly with values aligned with the axis corresponding to openness to change and growth (i.e., positive correlations with universalism, and selfdirection) versus conservation and self-protection (i.e., negative correlations with power and security). In terms of average absolute correlations with values, power, conformity, and universalism all exhibited slightly stronger correlations with personality factors than the other values. Finally, several correlations were greater than .40: power and honesty-humility (-.60), power and agreeableness (-.46), universalism and openness (.44), and universalism and honesty-humility (.40). The large correlations of universalism with two HEXACO factors (i.e., openness and honesty-humility) may be explained by its position in the values circumplex where it cross-loads on both openness to change and self-transcendence dimensions. 
Table 3

Zero-Order Correlations between HEXACO Personality Factors and Basic Personal Values

\begin{tabular}{|c|c|c|c|c|c|c|c|c|c|c|c|}
\hline & SD & ST & $\mathrm{HE}$ & $\mathrm{AC}$ & $\mathrm{PO}$ & $\mathrm{SE}$ & $\mathrm{CO}$ & TR & $\mathrm{BE}$ & UN & Mean Abs. $r$ \\
\hline Honesty-Humility & .08 & -.07 & -.19 & -.31 & -.60 & .07 & .23 & .17 & .29 & .40 & .24 \\
\hline Emotionality & -.23 & -.25 & .01 & .00 & .05 & .18 & .24 & .02 & -.01 & -.04 & .10 \\
\hline Extraversion & -.01 & .21 & .15 & .07 & -.20 & -.09 & -.18 & -.15 & .10 & .10 & .13 \\
\hline Agreeableness & -.15 & -.02 & -.08 & -.22 & -.46 & .00 & .26 & .20 & .08 & .32 & .18 \\
\hline Conscientiousness & -.07 & -.10 & -.23 & .11 & -.14 & .18 & .12 & .06 & .11 & .01 & .11 \\
\hline Openness & .27 & .32 & -.08 & -.06 & -.21 & -.19 & -.25 & -.19 & .04 & .42 & .20 \\
\hline Mean Absolute $r$ & .13 & .16 & .12 & .13 & .28 & .12 & .21 & .13 & .11 & .21 & .16 \\
\hline
\end{tabular}

Note. $N=1244 .|r| \geq .06$ is statistically significant $(p<.05)$. Correlations larger than .20 are bolded. Mean Abs. $r$ is the mean absolute correlation for the corresponding row or column. SD $=$ Self-direction, $\mathrm{ST}=$ Stimulation, $\mathrm{HE}=$ Hedonism, $\mathrm{AC}=$ Achievement, $\mathrm{PO}=$ Power, $\mathrm{SE}=$ Security, $\mathrm{CO}=$ Conformity, $\mathrm{TR}=$ Tradition, $\mathrm{BE}=$ Benevolence, $\mathrm{UN}=$ Universalism.

\section{Facet Correlations}

Table 4 presents correlations between observed HEXACO facets and the 10 basic values (i.e., zero-order correlations), and Table 5 presents correlations between personal values and the residualized facets (i.e., semi-partial correlations controlling for HEXACO factors). Substantial zero-order correlations commonly occurred in clusters within facets. For example, all honesty-humility facets were negatively related to power and all openness facets were positively related to self-direction. The largest average absolute facet correlations were observed for greedavoidance, unconventionality, gentleness, modesty, fairness, and altruism. In addition, within factors that tended to have weaker correlations with values, some facets stood out as relatively strong correlates with values (e.g., fearfulness in emotionality and diligence and prudence in conscientiousness). More generally, many of the residualized facet correlations with values were consistent with alignment of trait and value constructs: e.g., greed avoidance and power (-), altruism and universalism $(+)$; fearfulness and security $(+)$, diligence and achievement (+). 
Table 4

Correlations between HEXACO Personality Facets and Basic Personal Values

\begin{tabular}{|c|c|c|c|c|c|c|c|c|c|c|c|}
\hline & SD & ST & $\mathrm{HE}$ & $\mathrm{AC}$ & $\mathrm{PO}$ & SE & $\mathrm{CO}$ & TR & $\mathrm{BE}$ & $\mathrm{UN}$ & Mean Abs. $r$ \\
\hline H1: Sincerity & .09 & -.01 & -.14 & -.18 & -.41 & .05 & .11 & .10 & .16 & .26 & .15 \\
\hline H2: Fairness & -.07 & -.12 & -.24 & -.09 & -.34 & .12 & .26 & .12 & .17 & .22 & .18 \\
\hline H3: Greed-Avoidance & .19 & -.02 & -.13 & -.34 & -.54 & .00 & .12 & .14 & .27 & .41 & .22 \\
\hline H4: Modesty & .03 & -.07 & -.05 & -.31 & -.51 & .05 & .19 & .14 & .27 & .32 & .19 \\
\hline E1: Fearfulness & -.14 & -.34 & -.05 & .00 & .09 & .28 & .30 & .06 & -.11 & -.10 & .15 \\
\hline E2: Anxiety & -.13 & -.17 & -.03 & .05 & .17 & .11 & .15 & -.02 & -.02 & -.12 & .10 \\
\hline E3: Dependence & -.20 & -.08 & .11 & .02 & .02 & .03 & .08 & -.01 & -.01 & -.01 & .06 \\
\hline E4: Sentimentality & -.18 & -.10 & .01 & -.07 & -.19 & .08 & .14 & .01 & .14 & .17 & .11 \\
\hline X1: Social Self-Esteem & .04 & .09 & .08 & .03 & -.22 & -.02 & -.09 & -.08 & .12 & .07 & .08 \\
\hline X2: Social Boldness & .14 & .21 & -.01 & .12 & -.07 & -.09 & -.27 & -.19 & .12 & .11 & .13 \\
\hline X3: Sociability & -.20 & .15 & .27 & .04 & -.14 & -.07 & -.04 & -.10 & -.02 & .03 & .11 \\
\hline X4: Liveliness & -.01 & .23 & .11 & .02 & -.23 & -.08 & -.16 & -.09 & .12 & .11 & .12 \\
\hline Al: Forgiveness & -.10 & .07 & -.04 & -.15 &.- .33 & -.09 & .14 & .14 & .02 & .27 & .13 \\
\hline A2: Gentleness & -.24 & -.11 & -.06 & -.24 & -.36 & .06 & .34 & .27 & .02 & .22 & .19 \\
\hline A3: Flexibility & -.09 & -.02 & -.05 & -.17 & -.40 & .04 & .17 & .12 & .11 & .27 & .14 \\
\hline A4: Patience & -.04 & -.01 & -.10 & -.13 & -.34 & -.01 & .18 & .11 & .11 & .22 & .12 \\
\hline $\mathrm{C} 1$ : Organization & -.14 & -.09 & -.10 & .00 & -.07 & .22 & .14 & .06 & .03 & -.04 & .09 \\
\hline C2: Diligence & .04 & .12 & -.19 & .24 & -.13 & -.06 & -.11 & -.06 & .14 & .05 & .12 \\
\hline C3: Perfectionism & -.04 & -.14 & -.14 & .07 & -.05 & .14 & .16 & .05 & .05 & -.07 & .09 \\
\hline C4: Prudence & -.03 & -.18 & -.24 & .01 & -.18 & .19 & .16 & .12 & .12 & .09 & .13 \\
\hline O1: Aesthetic Appreciation & .16 & .15 & -.07 & -.10 & -.20 & -.10 & -.11 & -.13 & .07 & .39 & .15 \\
\hline $\mathrm{O} 2$ : Inquisitiveness & .18 & .18 & -.11 & -.04 & -.15 & -.09 & -.18 & -.09 & .05 & .31 & .14 \\
\hline O3: Creativity & .19 & .31 & -.04 & -.01 & -.16 & -.15 & -.21 & -.15 & .00 & .23 & .14 \\
\hline O4: Unconventionality & .33 & .37 & -.01 & -.04 & -.14 & -.28 & -.32 & -.23 & .01 & .36 & .21 \\
\hline I: Altruism & -.08 & -.11 & -.08 & -.17 & -.42 & .06 & .19 & .04 & .21 & .40 & .18 \\
\hline Mean Absolute $r$ & .12 & .14 & .10 & .11 & .23 & .10 & .17 & .11 & .10 & .19 & .14 \\
\hline
\end{tabular}

Note. Absolute correlations greater than .20 are bolded. $|\mathrm{r}| \geq .06$ is statistically significant $(p<$ $.05) ;|r| \geq .10$ is statistically significant $(p<.001)$. The "Mean Abs. $r$ " column indicates the mean absolute correlation over the 10 values. $\mathrm{SD}=$ Self-direction, $\mathrm{ST}=\mathrm{Stimulation}, \mathrm{HE}=\mathrm{Hedonism}$, $\mathrm{AC}=$ Achievement, $\mathrm{PO}=$ Power, $\mathrm{SE}=$ Security, $\mathrm{CO}=$ Conformity, $\mathrm{TR}=$ Tradition, $\mathrm{BE}=$ Benevolence, $\mathrm{UN}=$ Universalism. 
Table 5

Correlations between Residualized HEXACO Personality Facets and Basic Personal Values

\begin{tabular}{|c|c|c|c|c|c|c|c|c|c|c|c|}
\hline & SD & ST & $\mathrm{HE}$ & $\mathrm{AC}$ & $\mathrm{PO}$ & SE & $\mathrm{CO}$ & TR & $\mathrm{BE}$ & UN & Mean Abs r \\
\hline H1: Sincerity & -.01 & .02 & .02 & .06 & .05 & .02 & -.02 & -.01 & -.08 & -.08 & .04 \\
\hline H2: Fairness & -.13 & -.09 & -.16 & .11 & .16 & .05 & .14 & .03 & -.08 & -.07 & .10 \\
\hline H3: Greed-Avoidance & .13 & .02 & .01 & -.10 & -.13 & -.03 & -.06 & .01 & .08 & .11 & .07 \\
\hline H4: Modesty & .01 & .06 & .13 & -.08 & -.09 & -.04 & -.07 & -.03 & .09 & .04 & .06 \\
\hline E1: Fearfulness & .07 & -.15 & -.07 & .02 & .05 & .18 & .10 & .00 & -.15 & -.04 & .08 \\
\hline E2: Anxiety & .00 & .08 & .02 & .01 & .03 & -.07 & -.02 & -.05 & .04 & -.04 & .04 \\
\hline E3: Dependence & -.04 & .08 & .04 & .00 & -.04 & -.09 & -.05 & .03 & .01 & .04 & .04 \\
\hline E4: Sentimentality & -.04 & .01 & .02 & -.03 & -.05 & -.03 & -.05 & .02 & .13 & .05 & .04 \\
\hline X1: Social Self-Esteem & .09 & -.06 & .03 & .02 & .02 & .03 & -.02 & -.02 & -.01 & -.05 & .03 \\
\hline X2: Social Boldness & .11 & -.08 & -.23 & .07 & .07 & .06 & -.03 & -.03 & .11 & .05 & .08 \\
\hline X3: Sociability & -.15 & .06 & .16 & -.06 & -.06 & -.06 & .08 & .04 & -.12 & .00 & .08 \\
\hline X4: Liveliness & -.04 & .08 & .03 & -.03 & -.04 & -.02 & -.04 & .01 & .04 & -.01 & .03 \\
\hline A1: Forgiveness & .01 & .06 & -.03 & .02 & .02 & -.08 & -.04 & .02 & -.05 & .04 & .04 \\
\hline A2: Gentleness & -.14 & -.05 & .06 & -.09 & -.01 & .04 & .11 & .12 & -.05 & -.04 & .07 \\
\hline A3: Flexibility & .02 & .00 & .01 & .01 & -.05 & .06 & -.05 & -.05 & .03 & .03 & .03 \\
\hline A4: Patience & .10 & -.03 & -.03 & .05 & .03 & -.01 & -.01 & -.09 & .07 & -.04 & .05 \\
\hline $\mathrm{C} 1$ : Organization & -.09 & .04 & .10 & -.17 & -.03 & .09 & .04 & .03 & -.06 & .03 & .07 \\
\hline C2: Diligence & .04 & .13 & -.11 & .26 & .03 & -.17 & -.14 & -.07 & .06 & -.02 & .10 \\
\hline C3: Perfectionism & .02 & -.02 & .08 & -.06 & -.05 & -.05 & .08 & .03 & -.01 & -.04 & .04 \\
\hline C4: Prudence & .05 & -.16 & -.10 & .02 & .06 & .13 & .01 & .01 & .03 & .02 & .06 \\
\hline O1: Aesthetic Appreciation & -.07 & -.11 & .03 & -.05 & .00 & .03 & .07 & .01 & .04 & .04 & .05 \\
\hline O2: Inquisitiveness & -.08 & -.10 & -.04 & -.01 & .00 & .09 & .05 & .08 & .02 & .01 & .05 \\
\hline O3: Creativity & .02 & .08 & -.01 & .04 & .00 & .01 & -.01 & .00 & -.04 & -.11 & .03 \\
\hline O4: Unconventionality & .14 & .15 & .03 & .03 & .00 & -.15 & -.13 & -.11 & -.02 & .07 & .08 \\
\hline I: Altruism & -.01 & -.07 & .00 & -.02 & -.07 & .00 & .01 & -.04 & .11 & .15 & .05 \\
\hline Mean Abs r & .06 & .07 & .06 & .06 & .05 & .06 & .06 & .04 & .06 & .05 & .06 \\
\hline
\end{tabular}

Note. Absolute correlations greater than .10 are bolded. $|\mathrm{r}| \geq .06$ is statistically significant ( $p<$ $.05) ;|\mathrm{r}| \geq .10$ is statistically significant $(p<.001)$. The "mean abs $\mathrm{r}$ " column indicates the mean absolute correlation over the 10 values. $\mathrm{SD}=$ Self-direction, $\mathrm{ST}=$ Stimulation, $\mathrm{HE}=$ Hedonism, $\mathrm{AC}=$ Achievement, $\mathrm{PO}=$ Power, $\mathrm{SE}=$ Security, $\mathrm{CO}=$ Conformity, $\mathrm{TR}=$ Tradition, $\mathrm{BE}=$ Benevolence, $\mathrm{UN}=$ Universalism.

\section{Predictive Models}

We examined the incremental prediction of personality facets over factors for each value. Regression models were conducted predicting each value from the following predictor sets: (a) largest 
correlating factor, (b) largest correlating facet, (c) HEXACO factors without honesty-humility and openness, (d) HEXACO factors without honesty-humility, (e) all 6 HEXACO factors, and (f) all 25 HEXACO facets. Regressions using largest correlating factor or largest correlating facet involved using a single predictor. The choice of predictor varied across models depending on which factor or facet had the largest correlation for a given value. Largest correlating factor and facet models were included to address an alternative way of framing the factor-facet debate. Specifically, some researchers have focused on whether individual facets correlate more highly than individual factors. Further, the regressions were conducted with and without openness and honestyhumility because these two traits have been identified as the most predictive of values in past literature.

Table 6 shows adjusted multiple- $R$ for each set of personality predictors with each value as an outcome. On average, adjusted multiple $\mathrm{R}$ increased from .34 when only emotionality, extraversion, agreeableness and conscientiousness were included to .39 with the addition of openness, and from .39 to .45 with the addition of honestyhumility. Thus, both openness and honesty-humility increased prediction of values relative to the four and five factor models, but these increases were not drastically disproportionate to the baseline prediction. Facets increased multiple- $R$ in predicting values in raw terms from .45 to .53 (an average proportional increase of 0.20 ). In general, values with lower multiple- $R$ for factors tended to show greater increases in multiple- $R$ when facets were included. Power, universalism, and conformity were particularly well explained by personality facets. In contrast, benevolence, tradition, and security were less well explained by personality facets. 
Table 6

Adjusted Multiple Correlations for Regression Models Predicting Personal Values from HEXACO Personality Factors and Facets

\begin{tabular}{|c|c|c|c|c|c|c|c|c|c|c|c|c|}
\hline Predictors/Statistic & $k$ & SD & ST & $\mathrm{HE}$ & $\mathrm{AC}$ & $\mathrm{PO}$ & SE & $\mathrm{CO}$ & TR & $\mathrm{BE}$ & UN & Mean \\
\hline \multicolumn{13}{|l|}{ Baseline Models } \\
\hline Strongest factor $R_{\mathrm{adj}}$ & 1 & .27 & .32 & .23 & .31 & .60 & .19 & .26 & .20 & .29 & .42 & .31 \\
\hline Strongest facet $R_{\text {adj }}$ & 1 & .33 & .37 & .27 & .34 & .54 & .28 & .34 & .27 & .27 & .41 & .34 \\
\hline EXAC factors $R_{\mathrm{adj}}$ & 4 & .31 & .37 & .36 & .29 & .46 & .30 & .50 & .32 & .13 & .32 & .34 \\
\hline EXACO factors $R_{\text {adj }}$ & 5 & .42 & .45 & .38 & .30 & .48 & .34 & .54 & .36 & .12 & .51 & .39 \\
\hline \multicolumn{13}{|l|}{ Main Comparison } \\
\hline HEXACO factors $R_{\text {adj }}$ & 6 & .45 & .45 & .40 & .40 & .65 & .34 & .55 & .37 & .30 & .59 & .45 \\
\hline HEXACO facets $R_{\text {adj }}$ & 25 & .55 & .55 & .50 & .50 & .69 & .44 & .61 & .42 & .41 & .64 & .53 \\
\hline$\Delta R_{\text {adj }}$ HEXACO facets & & .09 & .10 & .11 & .10 & .04 & .11 & .06 & .05 & .12 & .05 & .08 \\
\hline Prop increase $R_{\text {adj }}$ facets & & 0.21 & 0.22 & 0.27 & 0.24 & 0.06 & 0.31 & 0.12 & 0.13 & 0.39 & 0.08 & 0.20 \\
\hline
\end{tabular}

Note. $R_{\text {adf }}$ for a given column is the adjusted multiple correlation coefficient for a linear regression of the corresponding personal value onto the indicated personality traits: Strongest factor/facet is the factor/facet that has the largest correlation with a given value; EXAC factors was HEXACO factors sans honesty-humility and openness. EXACO factors was HEXACO factors sans honesty-humility. $k$ is number of predictors in the corresponding regression model. $\Delta R_{\text {adj }}$ facets is the increase in multiple $\mathrm{r}$ for a regression model with facets compared to HEXACO factors: $R_{\text {adj }}^{\text {(facets) }}-R_{\text {adj }}^{\text {(factors) }}$. Proportion (i.e., Prop) increase $R_{\text {adj }}$ facets was $\left(R_{\text {adj }}^{\text {(facets) }}-R_{\text {adj }}^{\text {(factors) }}\right) / R_{\text {adj }}^{\text {(factors) }}$. The mean column indicates the mean value of the 10 values. $\mathrm{SD}=$ Self-direction, $\mathrm{ST}=$ Stimulation, $\mathrm{HE}=$ Hedonism, $\mathrm{AC}=$ Achievement, $\mathrm{PO}=$ Power, $\mathrm{SE}=$ Security, $\mathrm{CO}=$ Conformity, $\mathrm{TR}=$ Tradition, $\mathrm{BE}=$ Benevolence, $\mathrm{UN}=$ Universalism.

\section{Robustness Checks: Ipsatization, Age, and Gender}

While ipsatization is the standard way of scoring values, to understand the effect of ipsatization of values, several supplementary analyses were performed. First, the correlation between raw and ipsatized versions of the ten values were obtained; the mean correlation was .77 with a range of .60 to .88 . Thus, ipsatization has a moderate influence on the scoring of values. Second, the 60 cross-correlations between HEXACO factors and the 10 values were compared for ipsatized and raw values. The correlation between raw and ipsatized cross-correlations was .88 suggesting that the pattern of cross-correlations was quite similar 
across the two scoring methods. In addition, the mean absolute crosscorrelation was very similar for ipsatized (mean $r=.161$ ) and raw values (mean $r=.166$ ). Third, Table S5 in the online supplement presents the adjusted multiple $R$ for regressions predicting each value from different predictor sets. Table S5 is analogous to Table 6, except that raw values replace the ipsatized values of Table 6 . On average, adjusted multiple $R$ when predicting ipsatized values was slightly smaller than when predicting raw values (e.g., mean adjusted $R$ for HEXACO factors was .459 for raw and .449 for ipsatized values, and for HEXACO facets was .555 for raw and .531 for ipsatized values).

Further analyses were also performed to examine the effect of age and gender on the results. First, regression models were performed predicting each value and each personality factor from age and gender (female $=0$, male $=1$ ). In general, the effects of these demographic variables were small. Absolute standardized betas greater than .15 were obtained for age predicting hedonism (-0.27), benevolence (0.21), universalism (0.15), honesty-humility (0.19), and conscientiousness (0.15). Absolute standardized betas above .15 were obtained for gender predicting tradition (0.17) and emotionality (-0.32), where a positive coefficient indicates that males score higher on the value or trait. A second set of analyses were performed which compared the partial correlations between values and facets controlling for age and gender to the zero-order correlations between values and facets. The correlation of these 250 cross-correlations was .994 and the mean absolute crosscorrelation was almost identical using both approaches (i.e., .162 for partial and .160 for zero-order) suggesting that controlling for age and gender made almost no difference to the results.

\section{Discussion}

The present study examined the conceptual and empirical relations between personality traits and personal values focusing on the HEXACO model of broad and narrow traits. Results showed that the broad and narrow traits of the HEXACO model showed moderate to strong overlap with values. Building on research using the Big 5 factors (Parks-Leduc et al., 2015), the present study shows that the inclusion of honesty-humility and narrow traits leads to a greater overlap between personality and values. The strongest factor-level personality correlates with values were observed for honesty-humility and openness, although all factors showed meaningful correlations with values, and agreeableness in particular exhibited several notable correlations. The inclusion of narrow traits of the HEXACO model resulted in a modest but meaningful increase in overlap with values and highlighted the importance of facet-level analysis.

\section{HEXACO Factors and Values}

The results of this study build on the extensive literature using the Big 5 (Parks-Leduc et al., 2015) by showing that the overlap between 
traits and values is greater when using the HEXACO model. While the correlations of emotionality, extraversion, agreeableness, conscientiousness, and openness with values were of a similar magnitude to those observed in research using the Big 5, the inclusion of honestyhumility and narrow facets increased the overall overlap between personality and values. In summary, average adjusted multiple correlations were .39 for HEXACO without honesty-humility, .45 for HEXACO factors, and .53 for HEXACO facets. There was also substantial variability in the size of these personality-value multiple correlations over values. In particular, the values of power, conformity, and universalism had adjusted multiple correlations greater than .60 when predicted by HEXACO facets. Overall, openness and honesty-humility together appeared to capture relatively well two broad orthogonal axes of the circular motivation continuum: openness with the conservation versus openness to change axis and honesty humility with the self-enhancement versus self-transcendence axis. In general, the sizes of correlations are consistent with there being both a combination of construct overlap between personality traits and values and there being relatively strong causal interconnections between the constructs.

Theoretically, values are broad goals, motives, and priorities, and personality traits may be conceptualized as behavioral, cognitive, and affective tendencies. Further, in theory, values are stable, but not as stable as personality; values are also theorized to be influenced more by environment, whereas personality is often theorized to have a greater genetic cause. Personality traits are also conceptualized as latent both in the psychometric sense and in the deeper sense that there is an underlying constellation of biopsychosocial features that are leading to the manifest tendencies. While values are theoretically the internal reasons for a wide range of behavior, they also may reflect, and be influenced by, deep individual differences well captured by broad and narrow trait frameworks of personality.

Many measures and theories of personality traits are not optimized to be distinct from values. Parks-Leduc et al. (2015) recommend that researchers evaluate personality instruments at the itemlevel to assess whether items are value-laden. When we reviewed items from the HEXACO-PI-R, it became clear that the concept of value-laden is not always clear. First, some items include features indicating preference, liking, or enjoyment (e.g., "I'm interested in learning about the history and politics of other countries"). Such items involve the application of a value judgment. Thus, in a literal sense, they exist in the broad space that includes morality, aesthetics, preferences, and interests. However, they may only indirectly relate to the kinds of abstract values captured by Schwartz's framework. Second, there are items that describe behaviors that suggest a likely underlying value-based motivation. For example, agreeing with many of the items on the fairness facet (e.g., "I would never accept a bribe, even if it were very large") suggest that the 
person values fairness. However, it is also possible that fair behavior may be driven by other values such as a desire to avoid conflict, maintain a good reputation, or avoid trouble with authority. Finally, some HEXACO items are similar to how they would be phrased in a values-based instrument in that they focus on preferences and the object of the preference is abstract and aligns with broad values. In particular, many items on the greed avoidance facet ask about the importance of being very wealthy, possessing status symbols, and being socially recognized for having high-status (e.g., "Having a lot of money is not especially important to me."). As an aside, we also note that similar patterns of value-laden items can be seen on other common measures of personality such as the NEO-PI-R (Costa \& MacCrae, 1992).

Empirical results and conceptual analysis raise the question of whether it is appropriate to include honesty-humility in a model of personality. Conceptually, constructs like greed avoidance and modesty align closely with the self-transcendence and self-enhancement pole of fundamental values. Empirically, the honesty-humility factor and constituent facets had the largest average correlations with values. An item-level reading shows that many honesty-humility items are valueladen to various degrees. Some personality researchers have attempted to exclude traits related to character from personality measurement (Allport, 1937). However, there are several arguments for why the overlap of honesty-humility with values should not be used as a basis for critiquing its inclusion within a factorial personality model. First, the empirical overlap between honesty-humility and values is only slightly larger than the overlap of openness and values. Thus, to exclude traits that are closely aligned with values would also require the exclusion of openness from the Big 5 (and HEXACO). Second, while the HEXACO model of personality represents honesty-humility as a broad trait, many aspects of the trait are well reflected in the narrow traits of other dominant personality measures. In particular honesty-humility has strong correlations with agreeableness in the NEO-PI-R $(\mathrm{r}=.67$, Gaughan, Miller, \& Lynam, 2012) and strong overlap with the NEO-PI-R facets of modesty, straightforwardness, and tender-mindedness (see, for example, Miller, Gaughan, Maples, \& Price, 2011). It is also almost identical to the general factor underlying the dark triad (Lee \& Ashton, 2014), which is commonly recognized as an important set of narrow traits for predicting deviance-related behavior. Thus, we believe that the overlap between honesty-humility and values is largely a sign that the HEXACO model is providing a more complete picture of individual differences. Furthermore, while future research comparing the predictive validity of HEXACO and values is needed, results suggest that the HEXACO-PI-R reduces the need to have both a personality and values based measure in applied predictive validity settings. 


\section{HEXACO Facets and Values}

The inclusion of facets led to a modest but meaningful increase in the prediction of values. The adjusted multiple- $R$ increased by around $20 \%$ relative to a model with factors as predictors. A proportion increase of between $10 \%$ and $50 \%$ is quite similar to estimates obtained in the literature examining a range of other criteria (e.g., Anglim \& Grant, 2016; Horwood et al., 2015; Quevedo \& Abella, 2011). When looking at zero-order correlations, overlap with values often appears to mirror the factor-level correlations, although facets like greed-avoidance, gentleness, and unconventionality did stand out. However, after controlling for HEXACO factors, many facets still had several notable correlations with values. In several cases, it seemed that there was a particular facet in a domain that was more relevant to values than the others (e.g., fearfulness in emotionality, diligence in conscientiousness, and unconventionality in openness). Although we do not discuss each of these facet correlations in depth here, these facet-level correlates help to provide a more nuanced overview of the overlap between personality and values.

Overall, the ten basic values tended to align slightly better with narrow rather than broad traits. For instance, for seven out of the ten basic values, the largest zero-order correlation with personality was observed for a facet rather than a domain (e.g., self-direction and unconventionality $r=.33$ versus self-direction and openness $r=.27$ ). In particular, unconventionality was often the main element of openness that led to strong correlations with values. However, in contrast, average correlations with all 10 values tended to be similar between domains and the corresponding nested facet with the largest correlation (mean $r$ of .24 for honesty-humility versus .22 for greed-avoidance, .20 for openness versus .21 for unconventionality, .18 for agreeableness versus .19 for gentleness). Thus, it seems that broad traits align relatively well with broad dimensional aspects of values, whereas narrow traits are better aligned with specific values.

\section{Limitations and Future Research}

The present study has a few limitations that should be noted. First, as with the majority of the literature, the study used self-report to measure personality traits and personal values. Second, social desirability may have contributed to participants' responses thereby influencing their answers to items, although the confidential and low-stakes nature of the research should mostly limit this effect to self-deception. Third, the results are grounded in the particular personality and value frameworks and operationalized measures that were used. Care needs to be taken in generalizing results back to the Big 5 framework. Likewise, the measure of personal values focuses on ten abstract values identified as being fundamental and important across cultures; however, the domain of preferences, interests, morality, and aesthetics includes a much broader set of specific values. 


\section{Conclusion}

This study contributes to the understanding of the relationships between personality and values in several key ways. It is the first to extensively examine the role of personality facets and honesty-humility in relation to personal values. It is also novel in its systematic assessment of the incremental value of facet-level prediction in relation to values. The substantial sample size of over one thousand participants combined with the longer and more reliable 200 -item measure of personality facets allowed for the kinds of robust results that are especially important given both the multiplicities associated with comparing facets, and the use of many predictors in assessing incremental prediction of facets. Specifically, the study suggests that when honesty-humility and facets are included the overlap of personality with personal values goes from being moderate to more substantial. This is consistent with a framework of both overlapping constructs between values and traits, and with close interconnections.

\section{References}

Allport, G. W. (1937). Personality: A psychological interpretation. New York: Holt

Anglim, J., \& Grant, S. L. (2014). Incremental criterion prediction of personality facets over factors: Obtaining unbiased estimates and confidence intervals. Journal of Research in Personality, 53, 148-157.

Anglim, J., \& Grant, S. L. (2016). Predicting psychological and subjective well-being from personality: Incremental prediction from 30 facets over the big 5. Journal of Happiness studies, 17, 59-80.

Ashton, M. C., Lee, K., \& de Vries, R. E. (2014). The HEXACO Honesty-Humility, Agreeableness, and Emotionality Factors A Review of Research and Theory. Personality and Social Psychology Review, 18, 139-152.

Ashton, M. C., Lee, K., Perugini, M., Szarota, P., De Vries, R. E., Di Blas, L., . . De Raad, B. (2004). A six-factor structure of personality-descriptive adjectives: solutions from psycholexical studies in seven languages. Journal of Personality and Social Psychology, 86, 356-366.

Baron, H. (1996). Strengths and limitations of ipsative measurement. Journal of Occupational and Organizational Psychology, 69(1), 49-56.

Bilsky, W., \& Schwartz, S. H. (1994). Values and personality. European Journal of Personality, 8, $163-181$.

Blickle, G., Schlegel, A., Fassbender, P., \& Klein, U. (2006). Some personality correlates of business white-collar crime. Applied Psychology, 55, 220-233.

Costa Jr, P. T., \& McCrae, R. R. (1995). Domains and facets: Hierarchical personality assessment using the Revised NEO Personality Inventory. Journal of personality assessment, 64, 21-50.

Costa, P. T., \& MacCrae, R. R. (1992). Revised NEO personality inventory (NEO PI-R) and NEO five-factor inventory (NEO FFI): Professional manual: Psychological Assessment Resources.

DeYoung, C. G., Quilty, L. C., \& Peterson, J. B. (2007). Between facets and domains: 10 aspects of the Big Five. Journal of Personality and Social Psychology, 93(5), 880-896.

Dollinger, S. J., Leong, F. T., \& Ulicni, S. K. (1996). On traits and values: With special reference to openness to experience. Journal of Research in Personality, 30, 23-41.

Gaughan, E. T., Miller, J. D., \& Lynam, D. R. (2012). Examining the utility of general models of personality in the study of psychopathy: A comparison of the HEXACO-PI-R and NEO PI-R. Journal of personality disorders, 26(4), 513-523.

Haslam, N., Whelan, J., \& Bastian, B. (2009). Big Five traits mediate associations between values and subjective well-being. Personality and Individual Differences, 46, 40-42.

Horwood, S., Anglim, J., \& Tooley, G. (2015). Type D personality and the Five-Factor Model: A facet-level analysis. Personality and Individual Differences, 83, 50-54.

Lee, K., \& Ashton, M. C. (2004). Psychometric properties of the HEXACO personality inventory. Multivariate behavioral research, 39(2), 329-358. 
Lee, K., \& Ashton, M. C. (2006). Further assessment of the HEXACO Personality Inventory: two new facet scales and an observer report form. Psychological assessment, 18(2), 182-191.

Lee, K., \& Ashton, M. C. (2008). The HEXACO personality factors in the indigenous personality lexicons of English and 11 other languages. Journal of Personality, 76(5), 1001-1053.

Lee, K., \& Ashton, M. C. (2014). The dark triad, the big five, and the HEXACO model. Personality and Individual Differences, 67, 2-5.

Lee, K., Ashton, M. C., Morrison, D. L., Cordery, J., \& Dunlop, P. D. (2008). Predicting integrity with the HEXACO personality model: Use of self- and observer reports. Journal of Occupational and Organizational Psychology, 81, 147-167.

Lee, K., Ashton, M. C., Ogunfowora, B., Bourdage, J. S., \& Shin, K.-H. (2010). The personality bases of sociopolitical attitudes: The role of Honesty-Humility and Openness to Experience. Journal of Research in Personality, 44, 115-119.

Lee, K., Ashton, M. C., Pozzebon, J. A., Visser, B. A., Bourdage, J. S., \& Ogunfowora, B. (2009). Similarity and assumed similarity in personality reports of well-acquainted persons. Journal of Personality and Social Psychology, 96, 460-472.

Lee, K., Gizzarone, M., \& Ashton, M. C. (2003). Personality and the likelihood to sexually harass. Sex Roles, 49, 59-69.

Luk, C. L., \& Bond, M. H. (1993). Personality variation and values endorsement in Chinese university students. Personality and Individual Differences, 14, 429-437.

McCrae, R. R., \& Costa, P. T. (1987). Validation of the Five-Factor Model of personality across instruments and observers. Journal of Personality and Social Psychology, 52, 81-90.

Meade, A. W. (2004). Psychometric problems and issues involved with creating and using ipsative measures for selection. Journal of Occupational and Organizational Psychology, 77(4), 531-551.

Miller, J. D., Gaughan, E. T., Maples, J., \& Price, J. (2011). A comparison of agreeableness scores from the Big Five Inventory and the NEO PI-R: Consequences for the study of narcissism and psychopathy. Assessment, 18(3), 335-339.

Olver, J. M., \& Mooradian, T. A. (2003). Personality traits and personal values: a conceptual and empirical integration. Personality and Individual Differences, 35, 109-125.

Ones, D. S., \& Viswesvaran, C. (1996). Bandwidth-fidelity dilemma in personality measurement for personnel selection. Journal of Organizational Behavior, 17, 609-626.

Parks-Leduc, L., Feldman, G., \& Bardi, A. (2015). Personality Traits and Personal Values A Meta-Analysis. Personality and Social Psychology Review, 19, 3-29.

Paunonen, S. V., \& Ashton, M. C. (2001). Big five factors and facets and the prediction of behavior. Journal of Personality and Social Psychology, 81, 524-539.

Paunonen, S. V., Haddock, G., Forsterling, F., \& Keinonen, M. (2003). Broad versus narrow personality measures and the prediction of behaviour across cultures. European Journal of Personality, 17, 413-433.

Paunonen, S. V., \& Jackson, D. N. (2000). What is beyond the big five? Plenty! Journal of personality, 68, 821-835.

Pozzebon, J. A. (2006). Personality traits and personal values: an investigation into the importance of each in the prediction of behaviour. (Masters of Arts), Brock University. Retrieved from https://dr.library.brocku.ca/handle/10464/1411

Pozzebon, J. A., \& Ashton, M. C. (2009). Personality and values as predictors of self- and peer-reported behavior. Journal of Individual Differences, 30, 122-129.

Quevedo, R. J. M., \& Abella, M. C. (2011). Well-being and personality: Facet-level analyses. Personality and Individual Differences, 50, 206-211.

Roccas, S., Sagiv, L., Schwartz, S. H., \& Knafo, A. (2002). The big five personality factors and personal values. Personality and social psychology bulletin, 28, 789-801.

Rokeach, M. (1973). The nature of human values (Vol. 438). New York: Free Press

Saroglou, V., \& Muñoz-García, A. (2008). Individual differences in religion and spirituality: An issue of personality traits and/or values. Journal for the Scientific Study of Religion, 47, 83-101.

Schwartz, S. H. (1992). Universals in the content and structure of values: Theoretical advances and empirical tests in 20 countries. Advances in experimental social psychology, 25, 1-65.

Schwartz, S. H., Caprara, G. V., \& Vecchione, M. (2010). Basic personal values, core political values, and voting: A longitudinal analysis. Political Psychology, 31(3), 421-452.

Schwartz, S. H., Cieciuch, J., Vecchione, M., Davidov, E., Fischer, R., Beierlein, C., . . . Demirutku, K. (2012). Refining the theory of basic individual values. Journal of Personality and Social Psychology, 103, 663688. 
Sherman, R. A., \& Funder, D. C. (2009). Evaluating correlations in studies of personality and behavior: Beyond the number of significant findings to be expected by chance. Journal of Research in Personality, 43(6), 10531063.

Vecchione, M., Alessandri, G., Barbaranelli, C., \& Caprara, G. (2011). Higher-order factors of the big five and basic values: Empirical and theoretical relations. British Journal of Psychology, 102, 478-498.

Vernon, P. E., \& Allport, G. W. (1931). A test for personal values. The Journal of Abnormal and Social Psychology, 26(3), 231-248. 


\section{Online Supplementary Data}

\section{Supplementary analysis of HEXACO facets and Basic Values}

Exploratory factor analysis of the 25 HEXACO facets indicated good support for the theorized six factor solution. The scree plot showed clear support for a six-factor solution. The percentage of variance explained by the first ten unrotated components was $23,11,9,8,7,5,3,3$, 3, 3. After applying a promax rotation on the six-factor solution, all facets loaded maximally on their theorized factor, and there were only three facets with cross-loadings above .35 .

A multidimensional scaling (MDS) analysis of the ten basic values also broadly supported the circular structure proposed by Schwartz's (1992) theory. A two-dimensional non-metric MDS was employed with zero minus the correlation between corresponding values as the underlying distance measure. The best-fitting structure arranged the points in a circle consistent with theory with the exception that tradition had moved two spots clockwÍise to be between conformity and benevolence. 


\section{Internal Consistency and Descriptive Statistics}

Table S1

Descriptive Statistics and Cronbach's Alpha Internal Consistency for Basic Personal Values

\begin{tabular}{|c|c|c|c|c|}
\hline Variable & $\alpha$ & $M$ & $S D$ & Rank \\
\hline Self-direction & .68 & 5.00 & 0.56 & 2 \\
\hline Stimulation & .73 & 4.44 & 0.91 & 6 \\
\hline Hedonism & .76 & 4.48 & 0.85 & 5 \\
\hline Achievement & .63 & 4.52 & 0.84 & 4 \\
\hline Power & .82 & 3.51 & 0.81 & 9 \\
\hline Security & .74 & 4.37 & 0.78 & 7 \\
\hline Conformity & .84 & 3.94 & 0.93 & 8 \\
\hline Tradition & .72 & 3.30 & 1.26 & 10 \\
\hline Benevolence & .79 & 5.13 & 0.63 & 1 \\
\hline Universalism & .84 & 4.63 & 0.71 & 3 \\
\hline
\end{tabular}

Note. Means, SDs and Cronbach's alpha for personal values were computed using raw nonipsatized items. 
Table S2

Descriptive Statistics and Cronbach's Alpha Internal Consistency for HEXACO Personality

\begin{tabular}{|c|c|c|c|}
\hline Variable & $\alpha$ & $M$ & $S D$ \\
\hline \multicolumn{4}{|l|}{ HEXACO Factors } \\
\hline Honesty-humility & .89 & 3.70 & 0.46 \\
\hline Emotionality & .87 & 3.03 & 0.44 \\
\hline Extraversion & .92 & 3.72 & 0.49 \\
\hline Agreeableness & .90 & 3.16 & 0.46 \\
\hline Conscientiousness & .88 & 3.68 & 0.43 \\
\hline Openness to experience & .90 & 3.55 & 0.48 \\
\hline \multicolumn{4}{|l|}{ HEXACO Facets } \\
\hline H1: Sincerity & .77 & 3.64 & 0.57 \\
\hline H2: Fairness & .81 & 4.11 & 0.63 \\
\hline H3: Greed avoidance & .81 & 3.42 & 0.67 \\
\hline H4: Modesty & .78 & 3.64 & 0.58 \\
\hline E1: Fearfulness & .79 & 2.70 & 0.67 \\
\hline E2: Anxiety & .82 & 3.10 & 0.68 \\
\hline E3: Dependence & .78 & 2.84 & 0.60 \\
\hline E4: Sentimentality & .74 & 3.50 & 0.55 \\
\hline X1: Social self-esteem & .83 & 4.07 & 0.56 \\
\hline X2: Social boldness & .82 & 3.63 & 0.65 \\
\hline X3: Sociability & .85 & 3.41 & 0.69 \\
\hline X4: Liveliness & .83 & 3.79 & 0.60 \\
\hline A1: Forgiveness & .86 & 2.96 & 0.68 \\
\hline A2: Gentleness & .74 & 3.12 & 0.55 \\
\hline A3: Flexibility & .67 & 3.16 & 0.51 \\
\hline A4: Patience & .82 & 3.39 & 0.63 \\
\hline C1: Organization & .86 & 3.68 & 0.71 \\
\hline C2: Diligence & .80 & 3.84 & 0.57 \\
\hline C3: Perfectionism & .77 & 3.57 & 0.59 \\
\hline C4: Prudence & .77 & 3.63 & 0.54 \\
\hline O1: Aesthetic appreciation & .80 & 3.52 & 0.68 \\
\hline O2: Inquisitiveness & .80 & 3.75 & 0.65 \\
\hline O3: Creativity & .78 & 3.51 & 0.63 \\
\hline O4: Unconventionality & .73 & 3.42 & 0.52 \\
\hline I: Altruism & .76 & 4.00 & 0.50 \\
\hline
\end{tabular}

Note. Alpha for factors was computed using items not facet scores. 


\section{Scale Intercorrelations}

Table S3

Intercorrelations among HEXACO Factors

\begin{tabular}{|c|c|c|c|c|c|c|}
\hline & 1 & 2 & 3 & 4 & 5 & 6 \\
\hline 1. Honesty-humility & & -.03 & .19 & .42 & .28 & .14 \\
\hline 2. Emotionality & & & -.25 & -.23 & -.12 & -.15 \\
\hline 3. Extraversion & & & & .37 & .37 & .36 \\
\hline 4. Agreeableness & & & & & .24 & .18 \\
\hline 5. Conscientiousness & & & & & & .13 \\
\hline 6. Openness & & & & & & \\
\hline
\end{tabular}

Table S4

Intercorrelations among Ipsatized Personal Values

\begin{tabular}{|c|c|c|c|c|c|c|c|c|c|c|}
\hline & 1 & 2 & 3 & 4 & 5 & 6 & 7 & 8 & 9 & 10 \\
\hline 1. Self-direction & & .21 & -.09 & .01 & -.12 & -.26 & -.40 & -.33 & .08 & .16 \\
\hline 2. Stimulation & & & .23 & .01 & -.10 & -.49 & -.49 & -.33 & -.19 & .01 \\
\hline 3. Hedonism & & & & -.06 & -.05 & -.28 & -.24 & -.29 & -.17 & -.21 \\
\hline 4. Achievement & & & & & .35 & -.16 & -.30 & -.33 & -.14 & -.36 \\
\hline 5. Power & & & & & & .00 & -.16 & -.21 & -.27 & -.47 \\
\hline 6. Security & & & & & & & .29 & .17 & -.05 & -.10 \\
\hline 7. Conformity & & & & & & & & .30 & -.11 & -.05 \\
\hline 8. Tradition & & & & & & & & & -.03 & -.06 \\
\hline 9. Benevolence & & & & & & & & & & .19 \\
\hline 10. Universalism & & & & & & & & & & \\
\hline
\end{tabular}


Table S5

Adjusted Multiple Correlations for Regression Models Predicting Personal Non-Ipsatized Values from HEXACO Personality Factors and Facets

\begin{tabular}{lcccccccccccc}
\hline Predictors/Statistic & $k$ & SD & ST & HE & AC & PO & SE & CO & TR & BE & UN Mean \\
\hline Baseline Models & & & & & & & & & & & & \\
$\quad$ Strongest factor $R_{\text {adj }}$ & 1 & .36 & .33 & .28 & .27 & .48 & .33 & .27 & .18 & .35 & .44 & .33 \\
Strongest facet $R_{\text {adj }}$ & 1 & .31 & .34 & .38 & .39 & .56 & .31 & .37 & .22 & .35 & .49 & .37 \\
EXAC factors $R_{\text {adj }}$ & 4 & .34 & .34 & .35 & .40 & .37 & .42 & .49 & .24 & .43 & .41 & .38 \\
EXACO factors $R_{\text {adj }}$ & 5 & .46 & .41 & .35 & .40 & .38 & .43 & .51 & .26 & .43 & .56 & .42 \\
Main Comparison & & & & & & & & & & & & \\
HEXACO factors $R_{\text {adj }}$ & 6 & .46 & .42 & .40 & .49 & .57 & .43 & .51 & .29 & .44 & .58 & .46 \\
HEXACO facets $R_{\text {adj }}$ & 25 & .50 & .53 & .52 & .59 & .66 & .52 & .61 & .44 & .54 & .63 & .55 \\
$\Delta R_{\text {adj }}$ HEXACO facets & & .05 & .11 & .13 & .10 & .08 & .09 & .11 & .15 & .09 & .06 & .10 \\
Prop increase $R_{\text {adj }}$ facets & & 0.11 & 0.26 & 0.32 & 0.19 & 0.14 & 0.20 & 0.21 & 0.52 & 0.21 & 0.10 & 0.23 \\
\hline
\end{tabular}

Note. $R_{\text {adj }}$ for a given column is the adjusted multiple correlation coefficient for a linear regression of the corresponding personal value onto the indicated personality traits: Strongest factor is the factor or that has the largest correlation with a given value and strongest facet is the equivalent largest correlation at the facet-level; EXAC factors was HEXACO factors without honesty-humility and openness. EXACO factors was HEXACO factors without honestyhumility. $k$ is number of predictors in the corresponding regression model. $\Delta R_{\text {adj }}$ facets is the increase in multiple $\mathrm{r}$ for a regression model with facets compared to HEXACO factors: $R_{\text {adj }}^{\text {(facets) }}-R_{\text {adj }}^{\text {(factors) }}$. Proportion (i.e., Prop) change $R_{\text {adj }}$ facets was $\left(R_{\text {adj }}^{\text {(facets })}-R_{\text {adj }}^{\text {(factors })}\right) / R_{\text {adj }}^{\text {(factors })}$. The mean column indicates the mean value of the 10 values. $\mathrm{SD}=$ Self-direction, $\mathrm{ST}=$ Stimulation, $\mathrm{HE}=$ Hedonism, $\mathrm{AC}=$ Achievement, $\mathrm{PO}=$ Power, $\mathrm{SE}=$ Security, $\mathrm{CO}=$ Conformity, $\mathrm{TR}=$ Tradition, $\mathrm{BE}=$ Benevolence, $\mathrm{UN}=$ Universalism. 